\title{
Consecuencias de la neuromodulación endógena en el fenómeno de la desincronización de los ritmos sensorimotores en la enfermedad de Parkinson
}

\author{
del Castillo-Sobrino, M. Dolores; Serrano-Moreno, J. Ignacio y Rocon-de Lima, \\ Eduardo \\ Neural and Cognitive Engineering Group ( $\mathrm{gNeC}$ ) del Centro de Automática y Robótica (CAR) CSIC- \\ UPM, Ctra. Campo Real, km. 0,200, 28500 Arganda del Rey, Madrid, Spain, emails: \\ jignacio.serrano@,csic.es, e.rocon@csic.es, md.delcastillo@,csic.es
}

\begin{abstract}
This paper shows the results of a study of the desynchronization of the sensorimotor rhythms in Parkinson's disease (PD) after performing eight sessions of an endogenous neuromodulation intervention. Electrophysiological cortical activity is recorded while patients execute a wrist extension motor task before and after neuromodulation sessions. The goal of this study is included within a major objective aiming to compare the therapeutic efficacy of different neuromodulation strategies in PD patients.
\end{abstract}

Keywords: Parkinson's disease, sensorymotor rhytnms, neuromodulation.

\section{Resumen}

En este artículo se presentan los resultados de un estudio sobre los cambios que se producen en los ritmos sensorimotores de pacientes de la Enfermedad de Parkinson (EP) tras realizar ocho sesiones de neuromodulación endógena. La actividad electroencefalográfica analizada se registra mientras los pacientes realizan una tarea motora de extensión de muñeca antes y después de las sesiones de neuromodulación. El objetivo de este estudio se engloba dentro de un objetivo más amplio que busca comparar la eficacia terapéutica de diferentes técnicas de neuromodulación en EP.

Palabras clave: Enfermedad de Parkinson, ritmos sensorimotores, neuromodulación. 


\section{Introducción}

El movimiento voluntario es una seña de identidad del comportamiento humano. La planificación y ejecución de un movimiento es el resultado del procesamiento paralelo y distribuido de información entre diferentes áreas cerebrales corticales y subcorticales. La naturaleza oscilatoria, que gobierna la actividad cerebral, puede detectarse mediante señales periódicas, caracterizadas por un rango de frecuencia y una distribución topográfica, que a través de electroencefalografía (EEG). El estudio de estas oscilaciones en la corteza premotora, el área motora suplementaria, la corteza motora primaria y la corteza somatosensorial puede proporcionar conocimiento sobre aspectos inherentes a los procesos motores (Mostile, 2015).

La preparación y realización de un movimiento supone un evento que bloquea la actividad electroencefalográfica espontánea en determinadas bandas de frecuencia como consecuencia de cambios en algunos de los parámetros que controlan las oscilaciones de las redes neuronales implicadas en el mismo. Estos cambios relacionados con el movimiento se localizan en la corteza sensorimotora y se manifiestan mediante la desincronización (ERD, del inglés event-related-desynchronization) de la señal EEG, principalmente en las bandas de frecuencia alpha y beta. El fenómeno neurofisiológico de la desincronización conlleva una disminución de la potencia de la señal en esas bandas respecto de la potencia de la señal en un periodo previo al movimiento (Pfurtscheller, 1999). La desincronización del ritmo alpha es más destacado en la corteza contralateral sensorimotora, durante la preparación del movimiento, y se propaga simétricamente a ambos hemisferios cuando se inicia el movimiento. Las oscilaciones en la banda beta en esta área se bloquean durante la ejecución de un movimiento y con la estimulación somatosensorial (Neuper, 2006). En general, la desincronización de los ritmos alpha y beta refleja cambios en la actividad de las interacciones locales entre las neuronas e interneuronas de la corteza sensorimotora que afectan a las componentes en frecuencia de la señal electroencefalográfica espontánea.

Existe una correlación entre los parámetros y la morfología del fenómeno de la desincronización y la disfuncionalidad motora presente en trastornos del sistema nervioso central como el accidente cerebro vascular (ACV) y la enfermedad de Parkinson (EP) (Pfurtscheller, 1999) y su análisis puede conducir a mejorar el diagnóstico y la evolución de este tipo de enfermedades.

La EP se caracteriza por la progresiva degeneración de las neuronas dopaminérgicas de la sustancia negra que se acompaña de síntomas motores característicos como la lentitud de movimiento, la rigidez y la inestabilidad postural (Fahn, 2011). La medicación dopaminérgica produce normalmente una mejoría de los síntomas motores primarios pero 
algunos de ellos, como la dificultad en la marcha o la inestabilidad postural, apenas se modifica e incluso otros, como la disquinesia o la impulsividad, empeoran (Fahn, 2006). A pesar de ello, el tratamiento farmacológico es el mejor para esta enfermedad.

En la EP, los estudios realizados sobre el fenómeno de la desincronización de los ritmos sensorimotores apuntan a que, en la preparación del movimiento, éste está menos lateralizado sobre el área sensorimotora contralateral y se inicia con posterioridad a los controles sanos (Pfurtscheller, 1999). Por otro lado, muchos estudios neurofisiológicos llevados a cabo en este tipo de pacientes han descrito la presencia de una excesiva sincronización de los ritmos beta en el circuito formado por los ganglios basales, el tálamo y la corteza sensorimotora durante el reposo (Hutchison, 2014), (Sharott, 2014), que está asociada a la duración de la enfermedad y a la lentitud de los movimientos (Gulberti, 2015). Esta activación patológicamente alta, que muestra una escasa modulación durante la realización de un movimiento o tras la práctica repetitiva de tareas motoras (Moisello, 2015), puede normalizarse con el tratamiento dopaminérgico y la estimulación cerebral profunda así como con protocolos de regulación, como la neuromodulación endógena, de los patrones oscilatorios específicos motores (Esmail, 2014).

En (Heida, 2014) analizan mediante EEG la escasa desincronización del ritmo alpha en la corteza motora en pacientes con EP durante la observación de un movimiento en contraste con la desincronización bilateral experimentada por sujetos sanos. En pacientes con EP sin medicación se produce una exagerada desincronización del ritmo beta en la corteza motora suplementaria durante la realización de un movimiento, que se normaliza con el tratamiento dopaminérgico (Chung, 2018). En (Kondylis, 2016) se estudian las diferencias en la desincronización de los ritmos alpha y beta que produce una tarea motora entre pacientes con EP y con Temblor esencial a través de electrocorticografía.

Apenas existen en la literatura estudios sistemáticos en pacientes con EP sobre los parámetros de los ritmos sensorimotores mientras realizan una tarea motora, basados en el análisis de la actividad EEG, antes y después de la aplicación de intervenciones de neuromodulación.

En (Esmail, 2014) se realiza una revisión de varios trabajos de neuromodulación endógena en pacientes con EP y, en la mayoría de ellos, la evaluación de sus efectos se circunscribe al ámbito cinemático y motor (equilibrio, disquinesias, tiempos de reacción). El trabajo de (Fumuro, 2013) pone el foco en los potenciales evocados y en los potenciales corticales lentos en la corteza motora tras un procedimiento de neurorregulación de los potenciales lentos en el área motora central. Los autores de (Subramanian, 2011) evalúan la activación de áreas corticales premotoras y subcorticales, mediante resonancia magnética funcional, en una tarea de imaginación de movimiento. El trabajo de (Erickson, 2012) expone cómo la 
modulación exógena de los ritmos beta empleando estímulos auditivos produce una mejoría de la respuesta motora en EP.

En este trabajo se lleva a cabo el análisis de un conjunto de variables que caracterizan estructural y temporalmente el fenómeno neurofisiológico de la desincronización de los ritmos alpha y beta en la corteza sensorimotora en pacientes con EP. El estudio calcula estas variables antes y después de que los pacientes con EP se hayan sometido a una intervención de neuromodulación endógena.

\section{Neuromodulación de los ritmos sensorimotores}

El estudio aquí presentado se enmarca dentro de un proyecto de investigación cuyo objetivo general es el de evaluar y comparar la eficacia terapéutica de dos tipos de intervenciones de neuromodulación, una exógena, basada en la aplicación de estimulación magnética transcraneal repetitiva (rTMS) y, otra endógena, basada en mecanismos de autorregulación de la señal EEG a través de un entorno de realidad virtual. En ambos casos, se persigue modificar el sistema nervioso central, en base a la plasticidad neuronal, para producir mejoras de los síntomas motores mediante potenciación o inhibición de la actividad neuronal de la corteza sensorimotora.

Para ello, el proyecto ha contado con 40 pacientes con EP y 25 sujetos de control sanos. Los pacientes con EP se han dividido en un grupo de control y tres grupos experimentales. Cada grupo experimental ha llevado a cabo un tipo distinto de intervención de neuromodulación (neuromodulación endógena, neuromodulación exógena, una combinación de ambas). Las intervenciones de neuromodulación han tenido lugar en 8 sesiones a lo largo de dos semanas.

Antes y después de cada intervención de neuromodulación, los grupos experimentales han realizado una batería de tests clínicos motores, neurológicos, neuropsicológicos y de estabilidad. Además, se ha registrado su actividad electroencefalográfica durante la realización de dos tareas motoras (extensión de muñeca y unión repetitiva de los dedos índice y pulgar). Los grupos de control con EP y los sanos han sido valorados en una etapa inicial. Para el grupo de control con EP, también se ha llevado a cabo su valoración en una etapa posterior, dos semanas después.

En este artículo se presenta el análisis de la actividad oscilatoria presente en la señal EEG durante la realización de una de las tareas motoras en el grupo experimental sometido a la neuromodulación endógena y de las diferencias encontradas con el grupo de control sano. 


\section{Métodos y materiales}

\subsection{Población de estudio}

En este estudio han participado 11 pacientes con EP (4 mujeres, 7 hombres; diestros; edad media: $62.18 \pm 8.18$ años; tiempo desde el diagnóstico: $5.91 \pm 3.75$ años; Escala Unificada para la Enfermedad de Parkinson UPDRS: 15.55 \pm 7.29 , 5-32; dosis equivalente a levodopa (mg): 443 $\pm 218,100-710)$ y 25 sujetos sanos pareados por edad (6 mujeres, 19 hombres, edad media: 69.64 \pm 9.54 años). Ningún paciente con EP presentaba déficits cognitivos. Todos los pacientes realizaron este protocolo con el efecto de la medicación dopaminérgica, conocido como estado ON.

\subsection{Protocolo experimental y medición EEG}

Todos los sujetos permanecieron sentados durante la realización de una tarea motora que consistía en una tanda de 15 ensayos de extensión de muñeca, primero con la mano derecha y después con la mano izquierda, siguiendo un protocolo guiado a través de la pantalla de un ordenador portátil. En la Figura 1 se muestra el protocolo.

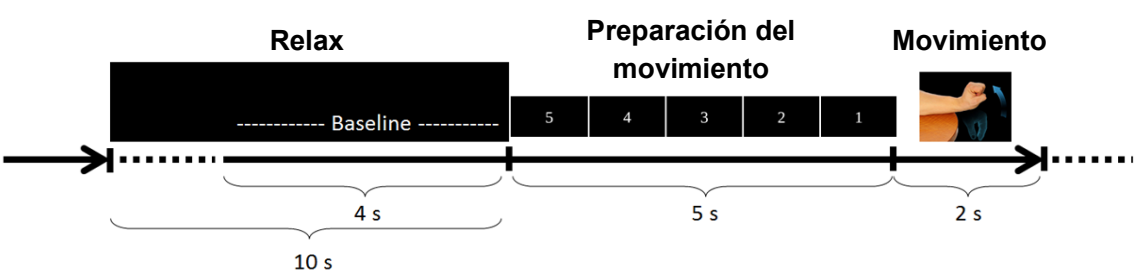

Fig. 1 Protocolo experimental guiado de la tarea motora de extensión de muñeca.

Durante esta tarea, se registraron las señales EEG de nueve canales situados en las áreas premotora, motora y somatosensorial (FC3, FCz, FC4, C3, Cz, C4, CP3, CPz y CP4 de acuerdo al sistema internacional 10-20) utilizando electrodos activos $\mathrm{Ag} / \mathrm{AgCl}$ (Acticap, Brain Products $\mathrm{GmbH}$, Germany). Los electrodos de tierra y referencia se ubicaron en $\mathrm{AFz}$ y $\mathrm{FCz}$, respectivamente. Las señales se amplificaron (BrainVision actiCHamp, Brain Products $\mathrm{GmbH}$, Germany) y se muestrearon a $250 \mathrm{~Hz}$. La adquisición de la señal EEG se llevó a cabo con el software NeuroRT Studio (Mensia Technologies SA, Paris, France). Los datos se han almacenado en un ordenador con Windows 7 (Microsoft Corporation, Washington, USA). 


\subsection{Desincronización de los ritmos alpha y beta}

En cada uno de los nueve electrodos considerados se estimó la densidad de potencia espectral (PSD) en ventanas solapadas de 1.5 segundos para las frecuencias entre 2 y $30 \mathrm{~Hz}$, con paso de $1 \mathrm{~Hz}$, empleando el método de Welch (ventanas de Hamming, $50 \%$ solapamiento). Se calculó la desincronización (ERD) de las señales en las bandas de frecuencia alpha $(7-13 \mathrm{~Hz})$ y beta $(13-30 \mathrm{~Hz})$, dentro de los períodos de Preparación del movimiento y de Movimiento (véase Figura 1), como el descenso estadísticamente significativo del valor de la potencia en esas ventanas respecto del valor de la potencia en la ventana temporal de Relax. El fenómeno ERD, para cada sujeto, canal, banda y lado del movimiento, se ha caracterizado mediante el valor medio en los 15 ensayos de un conjunto de variables en estos dos períodos.

Se ha analizado el fenómeno ERD en relación a tres segmentos temporales dentro de los dos períodos mencionados: a) ANT coincide con el período de Preparación del movimiento $(-5 \mathrm{~s}, 0)$; b) ANT-MOV, cuando el fenómeno se inicia en el período de Preparación del movimiento y se prolonga ininterrumpidamente al período de Movimiento; y c) MOV es período de Movimiento (0, 2s).

Las variables que caracterizan el fenómeno ERD, dependiendo del segmento temporal en que se localice son: 1) ERD Min, que representa el valor mínimo de ERD para cada uno de los segmentos temporales en los que se presente; 2) ERD Medio, que representa el valor medio de ERD para cada uno de los segmentos temporales en los que se presente; y 3) Porcentaje de tiempo que ocupa el fenómeno ERD dentro del segmento ANT o MOV; y 4) Duración en el tiempo del fenómeno ERD dentro del segmento ANT-MOV.

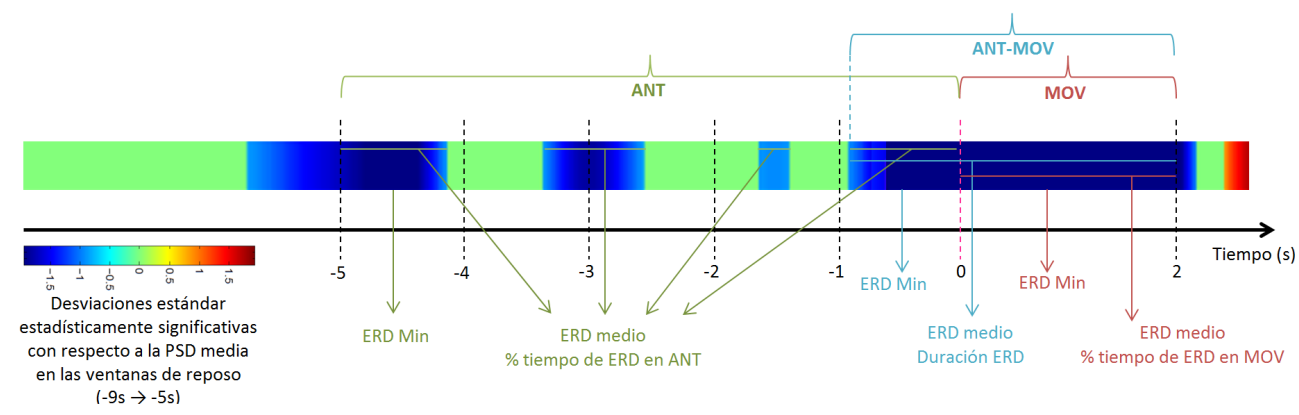

Fig. 2 Variables estructurales y temporales del fenómeno ERD durante la tarea de extensión de muñeca .

En la Figura 2 se muestra un ejemplo esquemático de la variación de la potencia de la señal en los tres segmentos temporales (ANT, ANT-MOV y MOV) y de las variables asociadas al fenómeno ERD para cada segmento para la tarea de extensión de muñeca. El valor de 
ERD en cada instante de tiempo es la potencia relativa a la potencia media en el período de Relax o basal.

Se han calculado también las diferencias entre las medias de cada una de las variables ERD del grupo experimental antes y después de la intervención de neuromodulación endógena y las medias de las variables ERD del grupo de control sano.

\subsection{Análisis estadístico}

Para cada variable extraída relacionada con el ERD se examinó la significación de la diferencia entre sus valores en el la etapa post-neuromodulación y pre-neuromodulación mediante un test $\mathrm{t}$ de medidas pareadas. Se utilizó el test de Levene para comprobar la igualdad de varianzas entre las poblaciones post y pre comparadas. En caso de no poder asumir igualdad de varianzas, se utilizó un test $\mathrm{t}$ de Welch para corregir dicha desigualdad. Todos los análisis estadísticos se realizaron mediante SPSS versión 25.0 (SPSS inc., Chicago, IL, USA). Los análisis se realizaron en un intervalo de confianza del 95\%. Los valores de significación $\mathrm{p}$ por debajo de 0,05 se consideraron estadísticamente significativos.

\section{Resultados}

Las Tablas 1 y 2 muestran los canales que han presentado cambios significativos de las variables del fenómeno ERD por la intervención de neuromodulación endógena en la mano derecha e izquierda, respectivamente. Se indica en negrita el nombre de la variable seguido entre paréntesis por la banda de frecuencia afectada. Debajo de cada variable, se presentan dos símbolos. El primero de ellos, $>$ ó <, representa si la variable ha incrementado o decrementado su valor, respectivamente, después de la intervención. El segundo, $\rightarrow$ ó $\leftarrow$, representa si el valor de la variable después de la intervención se aleja o se aproxima al valor medio de esa misma variable en los sujetos de control sanos.

Del análisis efectuado, se observa que se registran más cambios en el fenómeno ERD en ambas ventanas para la mano derecha, que es la mano dominante. Para esta mano, la mayor parte de los cambios se localizan en la corteza somatosensorial (CP3, CPz) y motora contralateral central (C3). En esta mano, todos los cambios en la corteza central $(\mathrm{Cz}, \mathrm{CPz}) \mathrm{e}$ ipsilateral (C4, CP4) se acercan a los valores medidos en los controles sanos. Estos cambios indican una menor desincronización en ambos ritmos alpha y beta, lo que apunta a una menor bilateralización de este fenómeno con la mano dominante tras la intervención de neruomodulación endógena. Los cambios se detectan en el período de Preparación del 
movimiento y se prolongan al período de Movimiento (ANT-MOV) y en el propio período de Movimiento (MOV).

Con respecto a la mano no dominante, la mayoría de los cambios tienen lugar en los segmentos temporales dentro del período de Preparación del movimiento (ANT y ANTMOV) y todos ellos indican una aproximación a los valores de los controles sanos. El tiempo de duración del fenómeno ERD se incrementa central y bilateralmente en ambas bandas de frecuencia en ese período. En la corteza prefrontal $(\mathrm{FCz})$, representando el área suplementaria motora, la desincronización en la banda beta aumenta acercándose a los controles sanos.

Tabla 1. Cambios estadísticamente significativos obtenidos en las variables neurofisiológicas relativas al fenómeno ERD en la mano DERECHA tras la intervención de neuromodulación endógena

\begin{tabular}{lcccccc}
\hline Mano derecha & C3 & CP3 & Cz & CPz & C4 & CP4 \\
\hline & $\begin{array}{c}\text { ERD Min } \\
\text { (alpha) }\end{array}$ & $\begin{array}{c}\text { ERD Medio } \\
\text { (alpha) }\end{array}$ & & & & \\
ANT-MOV & $<\rightarrow$ & & ERD Medio & & \\
& $>\leftarrow$ & ERD Min & & (beta) & & \\
& $($ alpha) & & $<\leftarrow$ & & \\
\hline & $<\rightarrow$ & & & & \\
MOV & \% ERD & \% ERD & \% ERD & ERD Medio & \% ERD \\
& & (beta) & (alpha) & (alpha) & (beta) & (alpha) \\
& & $<\rightarrow$ & $<\leftarrow$ & $<\leftarrow$ & $<\leftarrow$ & $<\leftarrow$ \\
\hline
\end{tabular}

Tabla 2. Cambios estadísticamente significativos obtenidos en las variables neurofisiológicas relativas al fenómeno ERD en la mano IZQUIERDA tras la intervención de neuromodulación endógena

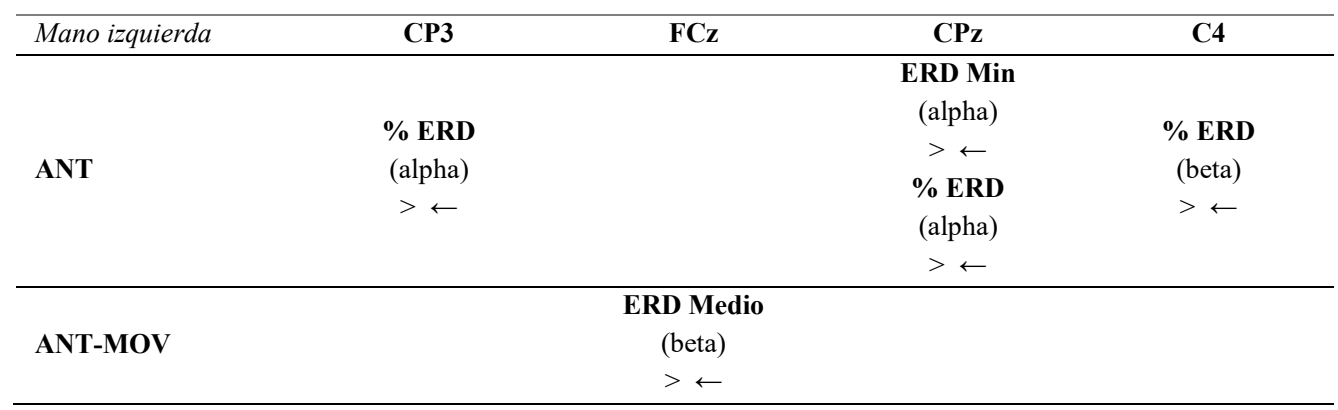




\section{Conclusiones}

La neuromodulación endógena efectivamente produce cambios sobre el fenómeno de la desincronización de los ritmos motores. En la mano dominante conduce a una menor desincronización relacionada con el movimiento en el hemisferio ipsilateral. En la mano no dominante produce un aumento en la duración de este fenómeno en el período de preparación del movimiento.

\section{Limitaciones del estudio}

El estudio realizado presenta limitaciones. La primera es que el tamaño de la muestra es relativamente pequeño. Los resultados podrían haber sido más significativos con un grupo mayor de pacientes con EP. La segunda limitación es la heterogeneidad que se presenta en la medicación, ya que los pacientes toman diferentes tipos de fármacos dependiendo de la estrategia terapéutica perseguida con el tratamiento. Todos los pacientes realizaron el estudio habiendo ingerido la medicación dopaminérgica pautada y encontrándose en el estado óptimo de influencia de la misma en las dos etapas del estudio, antes y después de la intervención de neuromodulación endógena, lo que necesariamente afecta a las variables neurofisiológicas medidas además de, posiblemente, a otros mecanismos corticales sensibles que no son tenidos en cuenta en este estudio.

\section{Agradecimientos}

El trabajo presentado forma parte de las investigaciones llevadas a cabo dentro de los proyectos NEUROMOD (DPI2015-68664-C4-1-R), MD (PIE-201650E055) y RoboCity2030-DIH-CM, Madrid Robotics Digital Innovation Hub, S2018/NMT-4331, financiado por "Programas de Actividades I + D en la Comunidad de Madrid" y cofinanciado por los Fondos Estructurales de la UE, habiendo sido financiado por ellos.

\section{Referencias}

Chung, JW., Burciu, RG., Oforic, E., Coombes, SA., Christou, EA., Okun, MS., Hess, CW., Vaillancour, DE. (2018). "Beta-band oscillations in the supplementary motor cortex are modulated by levodopa and associated with functional activity in the basal ganglia" en NeuroImage: Clinical, vol. 19, p. 559-571.

Erickson-Davis, CR., Anderson, JS., Wielinski, CL., Richter, SA., Parashos, SA. (2012). "Evaluation of neurofeedback training in the treatment of Parkinson's disease: A pilot study" en Journal of Neurotherapy, vol. 16, p. 4-11. 
Esmail, S., Linden, D. (2014). "Neural Networks and Neurofeedback in Parkinson's Disease" en NeuroRegulation, vol. 1, issue 3-4, p. 240-272.

Fahn, S. (2006). "A new look at levodopa based on the ELLDOPA study" en J. Neural Transm. Suppl. p. 419-26.

Fahn, S. (2011) “Classification of movement disorders”. En Mov. Disord. vol. 26, p. 947-57.

Fumuro, T., Matsuhashi, M., Mitsueda, T., Inouchi, M., Hitomi, T, Nakagawa, T., Brown, P. (2013). "Bereitschaftspotential augmentation by neuro-feedback training in Parkinson's disease" en Clinical Neurophysiology, vol. 124, p. 1398-1405.

Gulberti, A., Moll, C.K.E., Hamel, W., Buhmann, C., Koeppen, J.A. , Boelmans, K., Zittel, S., Gerloff, C., Westphal, M., Schneider, T.R., Engela, A.K. (2015). "Predictive timing functions of cortical beta oscillations are impaired in Parkinson's disease and influenced by L-DOPA and deep brain stimulation of the subthalamic nucleus" en NeuroImage: Clinical, vol. 9, p. 436-449.

Heida, T., Poppe, NR., de Vos, CC., van Putten, MJ., van Vugt, JP. (2014). "Event-related mu-rhythm desynchronization during movement observation is impaired in Parkinson's disease" en Clinical Neurophysiology, vol. 125, issue 9, p.1819-25.

Hutchison, WD., Dostrovsky, JO., Walters, JR., Courtemanche, R., Boraud, T., Goldberg, J., Brown, P. (2004). "Neuronal oscillations in the basal ganglia and movement disorders: evidence from whole animal and human recordings" en J. Neurosci. vol. 24, p. 9240-9243.

Kondylis, ED., Randazzo, MJ., Alhourani, A., Lipski, WJ., Wozny, TA., Pandya, Y., Ghuman, AS., Turner, RS., Crammond, DJ., Richardson, RM. (2016).” Movement-related dynamics of cortical oscillations in Parkinson's disease and essential tremor" en Brain, vol. 139, p. 2211-2223.

Moisello, C., Blanco, D., Lin, J., Panday, P., Kelly, SP., Quartarone, A. (2015). "Practice changes beta power at rest and its modulation during movement in healthy subjects but not in patients with Parkinson's disease" en Brain and Behavior, vol. 5, issue 10.

Mostile, G., Nicoletti, A., Dibilio, V., Luca, A., Pappalardo, I., Giuliano, L., Cicero, CE., Sciacca, G., Raciti, L., Contrafatto, D., Bruno, E., Sofia, V., Zappia, M. (2015). "Electroencephalographic lateralization, clinical correlates and pharmacological response in untreated Parkinson's disease" en Parkinsonism Relat Disord., vol. 21, issue 8, p. 948-53.

Neuper, C., Wörtz, M., Pfurtscheller, G. (2006). "ERD/ERS patterns reflecting sensorimotor activationand deactivation" en Neuper \& Klimesch (Eds.) Progress in Brain Research, vol. 159.

Pfurtscheller, G., Lopes da Silva, FH. (1999). "Event-related EEG/MEG synchronization and desynchronization: basic principles” en Clinical Neurophysiology, vol. 110, p.1842-1857.

Sharott, A., Gulberti, A., Zittel, S., Tudor Jones, AA., Fickel, U., Munchau, A., Koppen, JA., Gerloff, C., Westphal, M., Buhmann, C., Hamel, W., Engel, AK., Moll, CKE. (2014). “Activity parameters of subthalamic nucleus neurons selectively predictmotor symptom severity in Parkinson3s disease" en $J$. Neurosci., vol. 34, issue 18, p. 6273-6285.

Subramanian, L., Hindle, JV., Johnston, S., Roberts, MV., Husain, M., Goebel, R., Linden, D. (2011). "Real-time functional magnetic resonance imaging neurofeedback for treatment of Parkinson's disease" en Journal of Neuroscience, vol. 31, p. 16309-16317. 